\title{
Lattice path proofs of extended Bressoud-Wei and Koike skew Schur function identities
}

\author{
A. M. Hamel* \\ Department of Physics and Computer Science, \\ Wilfrid Laurier University, Waterloo, Ontario N2L 3C5, Canada \\ R. C. King ${ }^{\dagger}$ \\ School of Mathematics, University of Southampton, \\ Southampton SO17 1BJ, England
}

Submitted: Nov 29, 2010; Accepted: Feb 14, 2011; Published: Feb 21, 2011

Mathematics Subject Classification: 05E05

\begin{abstract}
Our recent paper [5] provides extensions to two classical determinantal results of Bressoud and Wei, and of Koike. The proofs in that paper were algebraic. The present paper contains combinatorial lattice path proofs.
\end{abstract}

Keywords: Schur functions, lattice paths

\section{Introduction}

Our recent paper [5] provides proofs of certain generalizations of two classical determinantal identities, one by Bressoud and Wei [1] and one by Koike [8]. Both of these identities are extensions of the Jacobi-Trudi identity, an identity that provides a determinantal representation of the Schur function. Here we provide lattice path proofs of these generalized idetities.

We give the barest of background details and notation, referring the reader instead to our earlier paper [5], and to Macdonald [10] or Stanley [11] for general symmetric function background knowledge.

*e-mail: ahamel@wlu.ca

†e-mail: r.c.king@soton.ac.uk 
Let $\mathcal{P}$ be the set of all partitions including the zero partition. Recall that in Frobenius notation each partition $\lambda=\left(\lambda_{1}, \lambda_{2}, \ldots\right) \in \mathcal{P}$ is written in the form

$$
\lambda=\left(\begin{array}{cccc}
a_{1} & a_{2} & \cdots & a_{r} \\
b_{1} & b_{2} & \cdots & b_{r}
\end{array}\right)
$$

with $a_{1}>a_{2}>\cdots>a_{r} \geq 0$ and $b_{1}>b_{2}>\cdots>b_{r} \geq 0$, where $a_{i}=\lambda_{k}-k$ and $b_{k}=\lambda_{k}^{\prime}-k$ for $k=1,2, \ldots, r$ with $\lambda^{\prime}$ the partition conjugate to $\lambda$. Here $r=r(\lambda)$, the rank of $\lambda$, which is defined to be the maximum value of $k$ such that $\lambda_{k} \geq k$. The partition $\lambda$ is said to have length $\ell(\lambda)=\lambda_{1}^{\prime}=b_{1}+1$ and weight $|\lambda|=\lambda_{1}+\lambda_{2}+\cdots=a_{1}+b_{1}+a_{2}+b_{2}+\cdots+a_{r}+b_{r}+r$. The case $r=0$ corresponds to the zero partition $\lambda=0=(0,0, \ldots)$ of length $\ell(\lambda)=0$ and weight $|\lambda|=0$.

For any integer $t$ let

$$
\mathcal{P}_{t}=\left\{\lambda=\left(\begin{array}{llll}
a_{1} & a_{2} & \cdots & a_{r} \\
b_{1} & b_{2} & \cdots & b_{r}
\end{array}\right) \in \mathcal{P} \mid a_{k}-b_{k}=t \quad \begin{array}{l}
\text { for } k=1,2, \ldots, r \\
\text { and } r=0,1, \ldots
\end{array}\right\}
$$

Here, as a matter of convention, it is to be assumed that the zero partition belongs to $\mathcal{P}_{t}$ for all integer $t$.

Let $m$ be a fixed positive integer and let $\mathbf{x}=\left(x_{1}, x_{2}, \ldots, x_{m}\right)$ be a sequence of $m$ indeterminates. Let $\lambda$ and $\sigma$ be partitions of lengths $\ell(\lambda), \ell(\sigma) \leq m$ such that $\sigma \subseteq \lambda$. We use the standard notation $h_{m}(\mathbf{x})$ to denote the complete homogeneous symmetric function of degree $m$ for $m>0$, with $h_{0}(\mathbf{x})=1$ and $h_{m}(\mathbf{x})=0$ for $m<0$. Further, $s_{\lambda}(\mathbf{x})$ and $s_{\lambda / \sigma}(\mathbf{x})$ denote the Schur function and skew Schur function specified by $\lambda$ and the pair $\lambda, \sigma$, respectively. Recall that the Jacobi-Trudi identity establishes the relationships:

$$
s_{\lambda}(\mathbf{x})=\left|h_{\lambda_{i}-i+j}(\mathbf{x})\right|
$$

and

$$
s_{\lambda / \sigma}(\mathbf{x})=\left|h_{\lambda_{i}-\sigma_{j}-i+j}(\mathbf{x})\right|,
$$

where the right-hand sides consist of $m \times m$ determinants, with $1 \leq i, j \leq m$, and the elements in the $i$ th row and $j$ th column have been displayed.

First Result: For all partitions $\lambda$ of length $\ell(\lambda) \leq m$, for all integers $t$ and any indeterminate $q$ we have

$$
\begin{aligned}
& \left|h_{\lambda_{i}-i+j}(\mathbf{x})+q \chi_{j>-t} h_{\lambda_{i}-i-j+1-t}(\mathbf{x})\right| \\
& =\sum_{\sigma \in \mathcal{P}_{t}}(-1)^{[|\sigma|-r(\sigma)(t+1)] / 2} q^{r(\sigma)} s_{\lambda / \sigma}(\mathbf{x}),
\end{aligned}
$$

where the determinant on the left is an $m \times m$ determinant, $\chi_{P}$ is the truth function [2] defined to be 1 if the proposition $P$ is true, and 0 otherwise, and the sum is over all partitions $\sigma$ in the set $\mathcal{P}_{t}$ with $r(\sigma) \leq m+\chi_{t<0} t$.

This is a generalization of the following result of Bressoud and Wei [1]: 
For all partitions $\lambda$ of length $\ell(\lambda) \leq m$ and all integers $t \geq-1$ one has

$$
\begin{aligned}
2^{(t-|t|) / 2} & \left|h_{\lambda_{i}-i+j}(\mathbf{x})+(-1)^{(t+|t|) / 2} h_{\lambda_{i}-i-j+1-t}(\mathbf{x})\right| \\
& =\sum_{\sigma \in \mathcal{P}_{t}}(-1)^{[|\sigma|+r(\sigma)(|t|-1)] / 2} s_{\lambda / \sigma}(\mathbf{x})
\end{aligned}
$$

where the determinant on the left is again an $m \times m$ determinant, and on the right the summation is over all partitions $\sigma$ in the set $\mathcal{P}_{t}$ of $\operatorname{rank} r(\sigma) \leq m$.

To go from (5) to (6), set $q=(-1)^{t}$ for all $t \geq 0$ and $q=1$ for $t=-1$. The factor $2^{(t-|t|) / 2}=2^{-1}$ when $t=-1$ compensates for the doubling of the entries in the first column of the determinant in (6) as compared to those in the corresponding column of (5).

If we allow two sets of variables, $\mathbf{x}=\left(x_{1}, x_{2}, \ldots, x_{m}\right)$ and $\mathbf{y}=\left(y_{1}, y_{2}, \ldots, y_{n}\right)$, then we can present our second result:

Second Result: First, let $m$ and $n$ be fixed positive integers, and let $\mathbf{x}=$ $\left(x_{1}, \ldots, x_{m}\right)$ and $\mathbf{y}=\left(y_{1}, \ldots, y_{n}\right)$. Then for all partitions $\lambda$ and $\mu$ of lengths $\ell(\lambda) \leq m$ and $\ell(\mu) \leq n$, for all integers $p$ and $q$, and any indeterminates $u$ and $v$, we have

$$
\begin{array}{|ccc}
h_{\mu_{n+1-i}+i-j}(\mathbf{y}) & \vdots & \chi_{j>n-q} u \\
\ldots & h_{\mu_{n+1-i}+i-j-q}(\mathbf{y}) \\
\ldots \\
\chi_{j \leq n+p} v h_{\lambda_{i-n}-i+j-p}(\mathbf{x}) & \vdots & h_{\lambda_{i-n}-i+j}(\mathbf{x})
\end{array} \mid
$$

where $r=r(\zeta), 1 \leq i, j \leq n+m$, and the $(n+m) \times(n+m)$ determinant is partitioned immediately after the $n$th row and $n$th column, and $\sigma+\tau$, for any pair of partitions $\sigma$ and $\tau$, signifies the partition whose $k$ th part is $\sigma_{k}+\tau_{k}$ for all $k[10, p 5]$.

It generalizes Koike's theorem [8]:

$$
\left|\begin{array}{c}
h_{\mu_{n+1-i}+i-j}(\mathbf{y}) \\
\cdots \\
h_{\lambda_{i-n}-i+j}(\mathbf{x})
\end{array}\right|=\sum_{\zeta \subseteq n^{m}}(-1)^{|\zeta|} s_{\lambda / \zeta}(\mathbf{x}) s_{\mu / \zeta^{\prime}}(\mathbf{y})
$$

For the two results (5) and (7) we will give combinatorial proofs based on lattice paths. In this connection, it is worth pointing out that the original Jacobi-Trudi identity can be given a very simple lattice path derivation as will be explained below. The lattice path technique was introduced by Gessel and Viennot [3, 4], finds full expression in Stembridge [12], and actually dates back to Karlin and McGregor [6, 7], and Lindström [9]. 


\section{Lattice Paths}

It is well-known that Schur functions can be defined using semistandard Young tableaux and in turn, all semistandard Young tableaux can be given a lattice path realisation (see, for example, [11, p. 343]). To this end, consider a square lattice and $m$-tuples of paths on this lattice, with the $i$ th path taking $\left(m-1+\lambda_{i}\right)$ successive unit steps either north or east from $P_{i}=(m+1-i, 1)$ to $Q_{i}=\left(m+1+\lambda_{i}-i, m\right)$ for $i=1,2, \ldots, m$. Let $\mathcal{T}^{\lambda}(m)$ be the set of semistandard Young tableaux of shape $\lambda$ and, similarly, $\mathcal{T}^{\lambda / \sigma}(m)$ be the set of semistandard Young tableaux of skew shape $\lambda / \sigma$. For each $T \in \mathcal{T}^{\lambda}(m)$ the corresponding $m$-tuple of paths is obtained by letting the entries read from left to right across the $i$ th row specify the heights of succesive eastward steps on the $i$ th path. It is not difficult to see that the semistandard nature of $T$ provides the necessary and sufficient conditions for the $m$ paths to be non-intersecting. The extension to the case of $T \in \mathcal{T}^{\lambda / \sigma}(m)$ is effected merely by defining new starting points $P_{i}=\left(m+1+\sigma_{i}-i, 1\right)$ for the $i$ th lattice path for $i=1,2, \ldots, m$.

For example, for $\lambda=(5,4,2)$ and $\sigma=(3,1)$ we have as possible examples of semistandard Young tableaux the following:

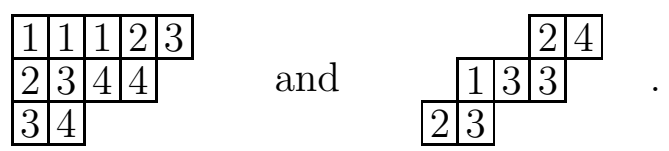

For $m=4$, the $m$-tuples of paths corresponding to the tableaux in (9) take the form

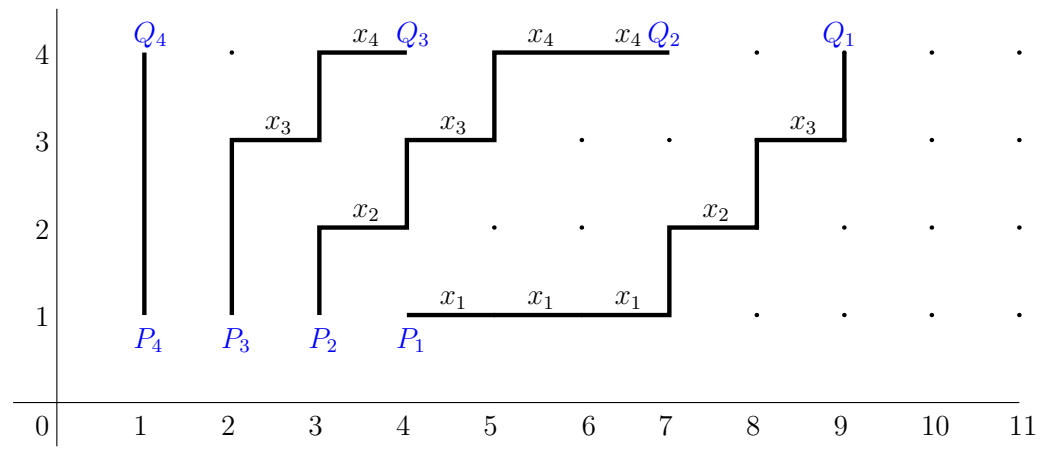

and

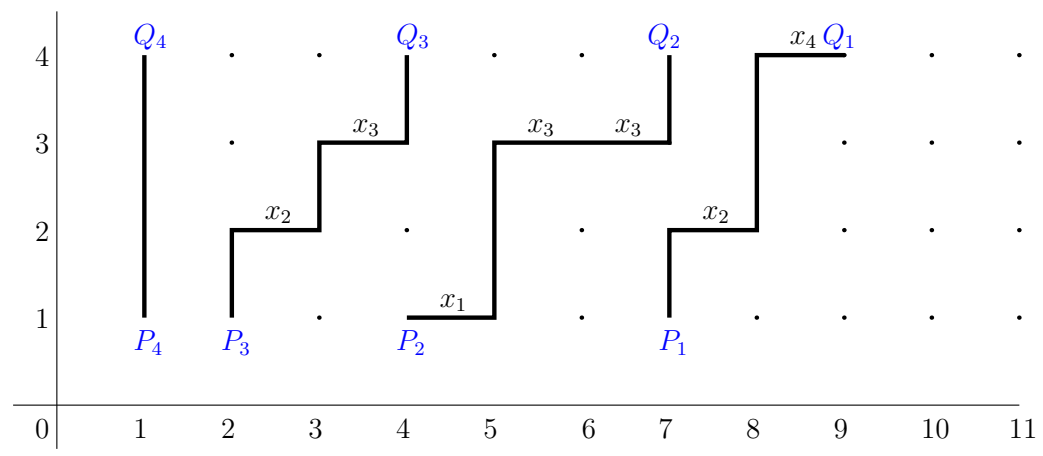

We denote the sets of all $m$-tuples of non-intersecting north-east lattice paths $L$ reaching a height no greater than $m$ by $\mathcal{L} \mathcal{P}^{\lambda}(m)$ and $\mathcal{L} \mathcal{P}^{\lambda / \sigma}(m)$, as appropriate. We now let 
each step east at height $k$ carry a weight $x_{k}$, with the total weight, $\mathbf{x}(L)$ of each $m$-tuple $L$ defined to be the product of the weights of all eastward steps. Thus our two 4-tuples illustrated in (10) and (11) are of weights $x_{1}^{3} x_{2}^{2} x_{3}^{3} x_{4}^{3}$ and $x_{1} x_{2}^{2} x_{3}^{3} x_{4}$, respectively.

The one-to-one correspondence between semistandard Young tableaux and $m$-tuples of non-intersecting north-east lattice paths implies that

$$
s_{\lambda}(\mathbf{x})=\sum_{L \in \mathcal{L} \mathcal{P}^{\lambda}(m)} \mathbf{x}(L) \text { and } s_{\lambda / \sigma}(\mathbf{x})=\sum_{L \in \mathcal{L} \mathcal{P}^{\lambda / \sigma}(m)} \mathbf{x}(L) .
$$

\section{$3 \quad$ Extended Bressoud-Wei identities}

The main result to be established here is the following:

Theorem 1 Let $m$ be a fixed positive integer, $\mathbf{x}=\left(x_{1}, x_{2}, \ldots, x_{m}\right)$ a sequence of indeterminates, and $\lambda=\left(\lambda_{1}, \lambda_{2}, \ldots, \lambda_{m}\right)$ a partition of length $\ell(\lambda) \leq m$. Then for all integers $t$ and any indeterminate $q$ we have

$$
\begin{aligned}
& \left|h_{\lambda_{i}-i+j}(\mathbf{x})+q \chi_{j>-t} h_{\lambda_{i}-i-j+1-t}(\mathbf{x})\right| \\
& =\sum_{\sigma \in \mathcal{P}_{t}}(-1)^{[|\sigma|-r(\sigma)(t+1)] / 2} q^{r(\sigma)} s_{\lambda / \sigma}(\mathbf{x}),
\end{aligned}
$$

where the determinant on the left is an $m \times m$ determinant.

Proof: We may write the expansion of the original determinant in the form

$$
\begin{aligned}
& \left|h_{\lambda_{i}-i+j}(\mathbf{x})+q \chi_{j>-t} h_{\lambda_{i}-i-j+1-t}(\mathbf{x})\right| \\
= & \sum_{\pi \in S_{n}}(-1)^{\pi} \prod_{i=1}^{m}\left(h_{\lambda_{i}-i+\pi(i)}(\mathbf{x})+q \chi_{\pi(i)>-t} h_{\lambda_{i}-i-\pi(i)+1-t}(\mathbf{x})\right),
\end{aligned}
$$

where for each $\pi$ the product on the right may be given a lattice path interpretation. To this end, let:

$$
\begin{array}{ll}
P_{i}=(m+1-i, 1) & \text { for } 1 \leq i \leq m ; \\
P_{i}^{\prime}=(m+t+i, 1) & \text { for } 1-\chi_{t<0} t \leq i \leq m ; \\
Q_{i}=\left(m+1-i+\lambda_{i}, m\right) & \text { for } 1 \leq i \leq m .
\end{array}
$$

It should be noted that the presence of the truth function $\chi_{t<0}$ ensures that the primed points $P_{i}^{\prime}$ all lie strictly to the east of the unprimed points $P_{i}$.

The product over $i$ on the right of (14) is then realised as a sum of contributions from all possible sets of $m$-tuples of north-east paths for which the $i$ th path goes from either $P_{\pi(i)}=(m+1-\pi(i), 1)$ or $P_{\pi(i)}^{\prime}=(m+t+\pi(i), 1)$ to $Q_{i}=\left(m+1+\lambda_{i}-i, m\right)$ for $i=1,2, \ldots, m$. Each step east at height $k$ carries weight $x_{k}$, and each path from $P_{\pi(i)}^{\prime}$ to $Q_{i}$, rather than from $P_{\pi(i)}$ to $Q_{i}$, carries an additional weight $q$. Each path from $P_{\pi(i)}$ to $Q_{i}$ contributes a monomial equal to the weight of the path to $h_{\lambda_{i}-i+\pi(i)}(\mathbf{x})$, and each one from $P_{\pi(i)}^{\prime}$ to $Q_{i}$ contributes a monomial equal to its weight to $h_{\lambda_{i}-i-\pi(i)+1-t}(\mathbf{x})$. 
For example, if $m=4, t=2, \lambda=(6,4,4,2)$ and $\pi=\left(\begin{array}{cccc}1 & 2 & 3 & 4 \\ 3^{\prime} & 1^{\prime} & 2 & 4\end{array}\right)$, with the primes indicating that the corresponding path starts from a $P_{j}^{\prime}$ rather than a $P_{j}$, then a possible 4-tuple of north-east paths takes the form

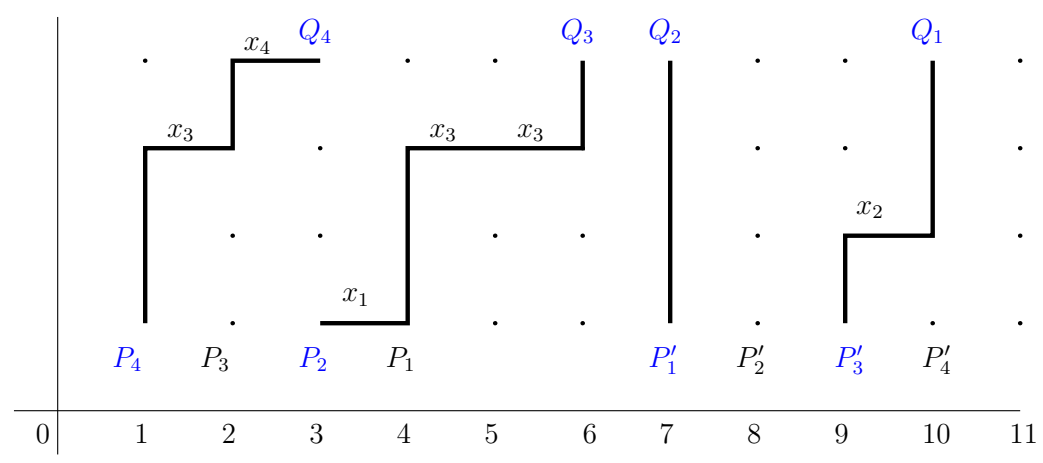

This gives a contribution $(-1)^{2+0}\left(q x_{2}\right)(q)\left(x_{1} x_{3}^{2}\right)\left(x_{3} x_{4}\right)=q^{2} x_{1} x_{2} x_{3}^{3} x_{4}$ to the product over $i$ in (14).

As usual, in the expansion of the determinant, a sign changing involution removes contributions from intersecting paths. For example, the following $m$-tuple involving intersecting paths arises in the case $m=4, \lambda=(6,6,6,4), t=2$ and $r=2$ :

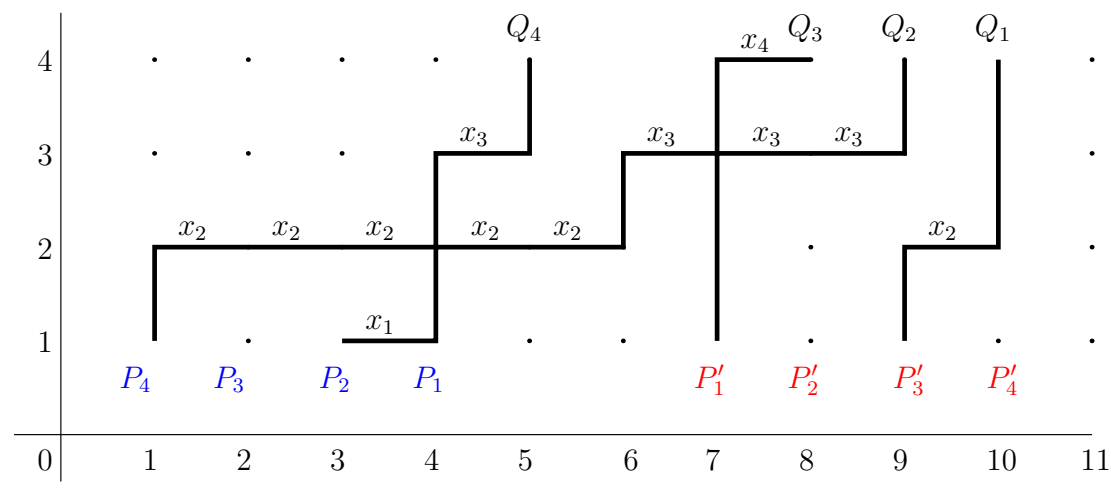

Such an $m$-tuple arises in the case of all four of the following permutations:

$$
\left(\begin{array}{cccc}
1 & 2 & 3 & 4 \\
3^{\prime} & 1^{\prime} & 2 & 4
\end{array}\right) ; \quad\left(\begin{array}{cccc}
1 & 2 & 3 & 4 \\
3^{\prime} & 1^{\prime} & 4 & 2
\end{array}\right) ; \quad\left(\begin{array}{cccc}
1 & 2 & 3 & 4 \\
3^{\prime} & 2 & 1^{\prime} & 4
\end{array}\right) ; \quad\left(\begin{array}{cccc}
1 & 2 & 3 & 4 \\
3^{\prime} & 4 & 1^{\prime} & 2
\end{array}\right) \text {. }
$$

As a matter of convention one may choose the sign changing involution to be the one generated by the transposition $(2,4)$ associated with the left-most point of intersection. Then contributions from the four permutations can be seen to cancel in pairs because of the presence of the factor $(-1)^{\pi}$ in the expansion (14).

If the paths in an $m$-tuple are to be non-intersecting then $\pi$ is necessarily such that:

$$
\begin{aligned}
& m \geq \pi(1)>\pi(2)>\cdots>\pi(r) \geq 1-\chi_{t>0} t ; \\
& 1 \leq \pi(r+1)<\pi(r+2)<\cdots<\pi(m) \leq m .
\end{aligned}
$$


To each such $\pi$ there corresponds a unique partition $\sigma \in \mathcal{P}_{t}$ of rank $r(\sigma)=r$. To see this it should be noted first that such permutations $\pi$ are in one-to-one correspondence with the partitions $\eta \subseteq\left(r^{m-r}\right)$ such that $\eta_{r}^{\prime} \geq-\chi_{t>0} t$. This correspondence is such that

$$
\pi=\left(\begin{array}{cccccccc}
1 & 2 & \cdots & r & r+1 & r+2 & \cdots & m \\
r+\eta_{1}^{\prime} & r-1+\eta_{2}^{\prime} & \cdots & 1+\eta_{r}^{\prime} & r+1-\eta_{1} & r+2-\eta_{r} & \cdots & m-\eta_{m-r}
\end{array}\right) .
$$

For given $\pi$, the partition $\eta$ may be constructed, in the spirit of Macdonald [10, p. 3] by labelling the consecutive boundary edges of $F^{\eta} \subseteq F^{\left(r^{m-r}\right)}$ with the integers $j=1,2, \ldots, m$, with the edge labelled $j$ either horizontal or vertical according as $\pi^{-1}(j)$ is either $\leq r$ or $>r$, as is illustrated later in (24) and (25).

Then the partitions $\eta \subseteq\left(r^{m-r}\right)$ with $\eta_{r}^{\prime} \geq-\chi_{t>0} t$ are in one-to one correspondence with the partitions $\sigma \in \mathcal{P}_{t}$ with $r(\sigma)=r$. This comes about because $F^{\sigma}$ may be constructed by appending $F^{\eta}$ and $F^{\eta^{\prime}+t^{r}}$ to the base and to the immediate right of $F^{r^{r}}$, as shown schematically by:

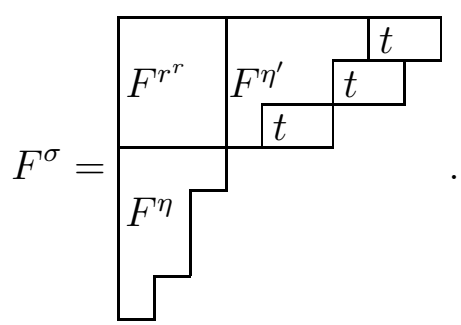

The condition $\eta_{r}^{\prime} \geq-\chi_{t>0} t$ is just what is required in order to ensure that $\sigma$ is indeed a partition for all $t$, including negative values.

It then follows that

$$
\pi=\left(\begin{array}{cccccccc}
1 & 2 & \cdots & r & r+1 & r+2 & \cdots & m \\
\sigma_{1}-t & \sigma_{2}+1-t & \cdots & \sigma_{r}-r+1-t & r+1-\sigma_{r+1} & r+2-\sigma_{r+2} & \cdots & m-\sigma_{m}
\end{array}\right) .
$$

so that

$$
\pi(i)= \begin{cases}\sigma_{i}-i+1-t & \text { for } i=1,2, \ldots, r \\ i-\sigma_{i} & \text { for } i=r+1, r+2, \ldots, m .\end{cases}
$$

For example, in the following two cases, both with $r=2$ but the first with $t=2$ and the second with $t=-2$, we have

$$
\pi=\left(\begin{array}{cccc}
1 & 2 & 3 & 4 \\
3^{\prime} & 1^{\prime} & 2 & 4
\end{array}\right) \quad \Longleftrightarrow \quad F^{\eta}=\sqrt{4_{3^{3^{\prime}}} 2^{1^{\prime}}} \quad \Longleftrightarrow \quad F^{\sigma}=\stackrel{\square+}{\square}
$$

and

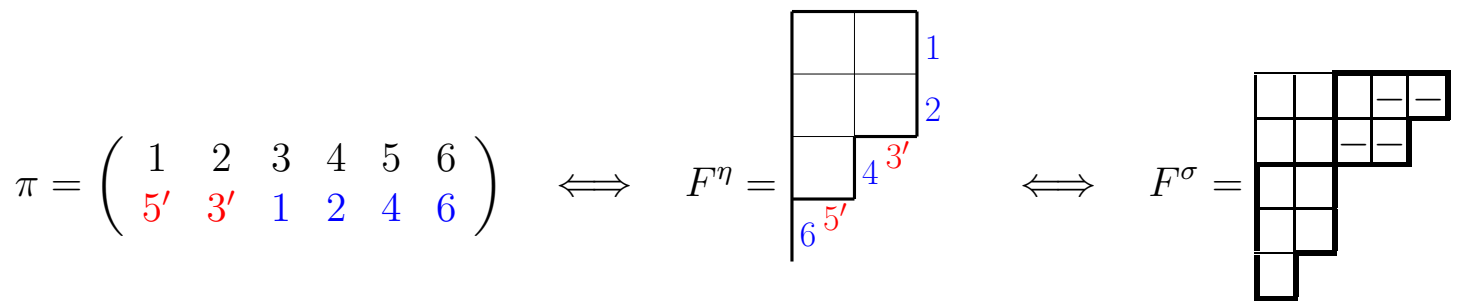


where the boxes containing + are to be included and those containing - are to be excluded.

Returning to our lattice paths, if we designate the eastward distance from $X$ to $Y$ by $|X Y|$, then $\left|P_{i} Q_{i}\right|=\lambda_{i}$ for all $i=1, \ldots, m,\left|P_{i} P_{\pi(i)}^{\prime}\right|=i+\pi(i)+t-1=\sigma_{i}$ for $i=1, \ldots, r$ and $\left|P_{i} P_{\pi(i)}\right|=i-\pi(i)=\sigma_{i}$ for $i=r+1, \ldots, m$. Hence the number of horizontal steps on the $i$ th path from $P_{\pi(i)}^{\prime}$ to $Q_{i}$ is $\lambda_{i}-\sigma_{i}$ for $i=1, \ldots, r$ and from $P_{\pi(i)}$ to $Q_{i}$ is $\lambda_{i}-\sigma_{i}$ for $i=r+1, \ldots, m$. The $i$ th path monomial of degree $\lambda_{i}-\sigma_{i}$ may then be interpreted as the contribution arising from the $i$ th row of an $s_{\lambda / \sigma}(\mathbf{x})$ skew semistandard tableau for all $i=1,2, \ldots, m$. It is the non-intersecting nature of the $m$-tuple of paths that guarantees that the tableau is skew semistandard.

Moreover, in Frobenius notation

$$
\sigma=\left(\begin{array}{cccc}
\pi(1)-1+t & \pi(2)-1+t & \cdots & \pi(r)-1+t \\
\pi(1)-1 & \pi(2)-1 & \cdots & \pi(r)-1
\end{array}\right)
$$

so that $\sigma \in \mathcal{P}_{t}$ with $|\sigma|=2(\pi(1)+\cdots+\pi(r)-r)+r(t+1)$. Since $(-1)^{\pi}=(-1)^{\pi(1)+\cdots+\pi(r)-r}$ we have, as required,

$$
\left|h_{\lambda_{i}-i+j}(\mathbf{x})+q \chi_{j>-t} h_{\lambda_{i}-i-j+1-t}(\mathbf{x})\right|=\sum_{\sigma \in \mathcal{P}_{t}}(-1)^{[|\sigma|-r(t+1)] / 2} q^{r} s_{\lambda / \sigma}(\mathbf{x}) .
$$

This completes the combinatorial proof of Theorem 1.

QED

For example, if $m=4, t=2, \lambda=(6,4,4,2), r=2$ and $\pi=\left(\begin{array}{cccc}1 & 2 & 3 & 4 \\ 3^{\prime} & 1^{\prime} & 2 & 4\end{array}\right)$, then from $(24) \sigma=(5,4,1)=\left(\begin{array}{ll}4 & 2 \\ 2 & 0\end{array}\right) \in \mathcal{P}_{2}$. The correspondence between non-intersecting 4-tuples of lattice paths and skew semistandard tableaux is then exemplified by

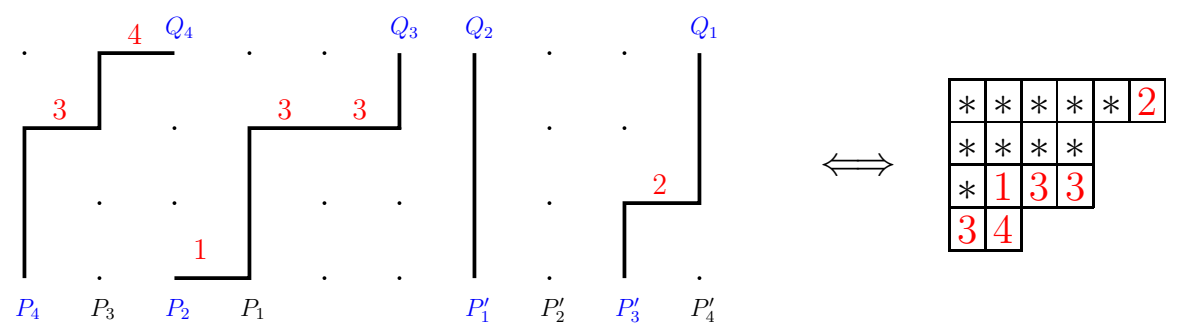

Similarly, if $m=6, t=-2, \lambda=(5,4,4,3,3,2)$ and $\pi=\left(\begin{array}{cccccc}1 & 2 & 3 & 4 & 5 & 6 \\ 5^{\prime} & 3^{\prime} & 1 & 2 & 4 & 6\end{array}\right)$, then from $(25) \sigma=(3,2,2,2,1)=\left(\begin{array}{ll}2 & 0 \\ 4 & 2\end{array}\right) \in \mathcal{P}_{-2}$, and the one-to-one correspondence between non-intersecting 6-tuples of lattice paths and skew semistandard tableaux is illustrated by: 


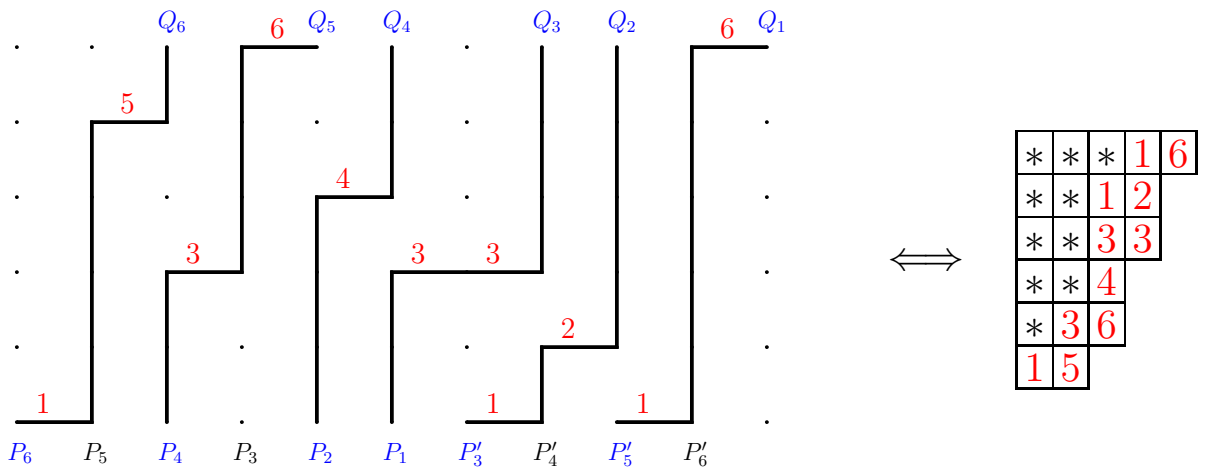

\section{Skew extension of the Koike identity}

Our second main result takes the form:

Theorem 2 For fixed positive integers $m$ and $n$, let $\mathbf{x}=\left(x_{1}, \ldots, x_{m}\right)$ and $\mathbf{y}=\left(y_{1}, \ldots, y_{n}\right)$ be two sequences of indeterminates, and let $\lambda$ and $\mu$ be a pair of partitions of lengths $\ell(\lambda) \leq m$ and $\ell(\mu) \leq n$. Then for each pair of integers $p$ and $q$, and any indeterminates $u$ and $v$, we have

$$
\begin{aligned}
& \left|\begin{array}{ccc}
h_{\mu_{n+1-i}+i-j}(\mathbf{y}) & \vdots & \chi_{j>n-q} u h_{\mu_{n+1-i}+i-j-q}(\mathbf{y}) \\
\ldots & \ldots \\
\chi_{j \leq n+p} v h_{\lambda_{i-n}-i+j-p}(\mathbf{x}) & \vdots & h_{\lambda_{i-n}-i+j}(\mathbf{x})
\end{array}\right| \\
& =\sum_{\zeta \subseteq n^{m}}(-1)^{|\zeta|}(u v)^{r} s_{\lambda /\left(\zeta+p^{r}\right)}(\mathbf{x}) s_{\mu /\left(\zeta^{\prime}+q^{r}\right)}(\mathbf{y})
\end{aligned}
$$

where $r=r(\zeta)$ and the $(n+m) \times(n+m)$ determinant is partitioned immediately after the nth row and nth column. If $\zeta \subseteq\left(n^{m}\right)$ is given in Frobenius notation by $\zeta=\left(\begin{array}{llll}a_{1} & a_{2} & \cdots & a_{r} \\ b_{1} & b_{2} & \cdots & b_{r}\end{array}\right)$, with $n>a_{1}>a_{2}>\cdots>a_{r}$ and $m>b_{1}>b_{2}>\cdots>b_{r}$, then:

$$
\zeta+p^{r}=\left(\begin{array}{cccc}
a_{1}+p & a_{2}+p & \cdots & a_{r}+p \\
b_{1} & b_{2} & \cdots & b_{r}
\end{array}\right)
$$

and

$$
\zeta^{\prime}+q^{r}=\left(\begin{array}{cccc}
b_{1}+q & b_{2}+q & \cdots & b_{r}+q \\
a_{1} & a_{2} & \cdots & a_{r}
\end{array}\right)
$$

with $a_{r} \geq \max \{0,-p\}$ and $b_{r} \geq \max \{0,-q\}$.

Proof: The determinant that is the subject of Theorem 2 can be expressed in the following form and expanded as shown 


$$
\begin{aligned}
& \left|\begin{array}{ccc}
\chi_{j \leq n} h_{\mu_{n+1-i}+i-j-d_{j}}(\mathbf{y}) & \vdots & u \chi_{j>n-q} h_{\mu_{n+1-i}+i-j-d_{j}}(\mathbf{y}) \\
\ldots & & \cdots \\
v \chi_{j \leq n+p} h_{\lambda_{i-n}-i+j-c_{j}}(\mathbf{x}) & \vdots & \chi_{j>n} h_{\lambda_{i-n}-i+j-c_{j}}(\mathbf{x})
\end{array}\right| \\
& =\sum_{\pi \in S_{n+m}}(-1)^{\pi} \prod_{i=1}^{n}\left(\chi_{\pi(i) \leq n}+u \chi_{\pi(i)>n-q}\right) h_{\mu_{n+1-i}+i-\pi(i)-d_{\pi(i)}}(\mathbf{y}) \\
& \prod_{i=n+1}^{n+m}\left(v \chi_{\pi(i) \leq n+p}+\chi_{\pi(i)>n}\right) h_{\lambda_{i-n}-i+\pi(i)-c_{\pi(i)}}(\mathbf{x})
\end{aligned}
$$

where

$$
c_{j}=\left\{\begin{array}{ll}
0 & \text { if } j>n ; \\
p & \text { if } j \leq n,
\end{array} \quad \text { and } \quad d_{j}= \begin{cases}0 & \text { if } j \leq n \\
q & \text { if } j>n\end{cases}\right.
$$

In order to give each term on the right a lattice path interpretation it is convenient to let:

$$
\begin{array}{ll}
S_{i}=(1-i, 1) & \text { for } 1 \leq i \leq n ; \\
S_{i}^{\prime}=(1-i-q, 1) & \text { for } n-\chi_{q<0} q<i \leq m+n \\
P_{i}^{\prime}=(m+n+1-i+p, 1) & \text { for } 1 \leq i \leq n+\chi_{p<0} p ; \\
P_{i}=(m+n+1-i, 1) & \text { for } n<i \leq m+n,
\end{array}
$$

and

$$
\begin{array}{ll}
R_{i}=\left(1-i-\mu_{n+1-i}, n\right) & \text { for } 1 \leq i \leq n: \\
Q_{i}=\left(m+n+1-i+\lambda_{i-n}, m\right) & \text { for } n<i \leq m+n .
\end{array}
$$

Now we return to the sum over $\pi \in S_{n+m}$ in (33). Each $\pi$ defines a set of $(n, m)$-tuples of lattice paths. For $i=n+1, n+2, \ldots, n+m$ the $i$ th north-east path goes from either $P_{\pi(i)}^{\prime}=(m+n+1+p-\pi(i), 1)$ or $P_{\pi(i)}=(m+n+1-\pi(i), 1)$ to $Q_{i}=\left(m+n+1-i+\lambda_{i-n}, m\right)$. Each step east at height $k$ carries weight $x_{k}$, with an additional factor of $u$ if the path starts from $P_{\pi(i)}^{\prime}$ as opposed to $P_{\pi(i)}$. For $i=1,2, \ldots, n$ the $i$ th north-west path goes from either $S_{\pi(i)}=(1-\pi(i), 1)$ or $S_{\pi(i)}^{\prime}=(1-q-\pi(i), 1)$ to $R_{i}=\left(1-i-\mu_{n+1-i}, n\right)$. In this case each step west at height $k$ carries weight $y_{k}$, with an additional factor of $v$ if the path starts from $S_{\pi(i)}^{\prime}$ as opposed to $S_{\pi(i)}$.

Typically, in the case, $m=3, n=4, p=-2, q=-1, \lambda=(5,3,2), \mu=(4,3,2,2)$ and

$$
\pi=\left(\begin{array}{lllllll}
1 & 2 & 3 & 4 & 5 & 6 & 7 \\
2 & 3 & 4 & 7 & 1 & 5 & 6
\end{array}\right)
$$

one such $(n, m)$-tuple of lattice paths takes the form 


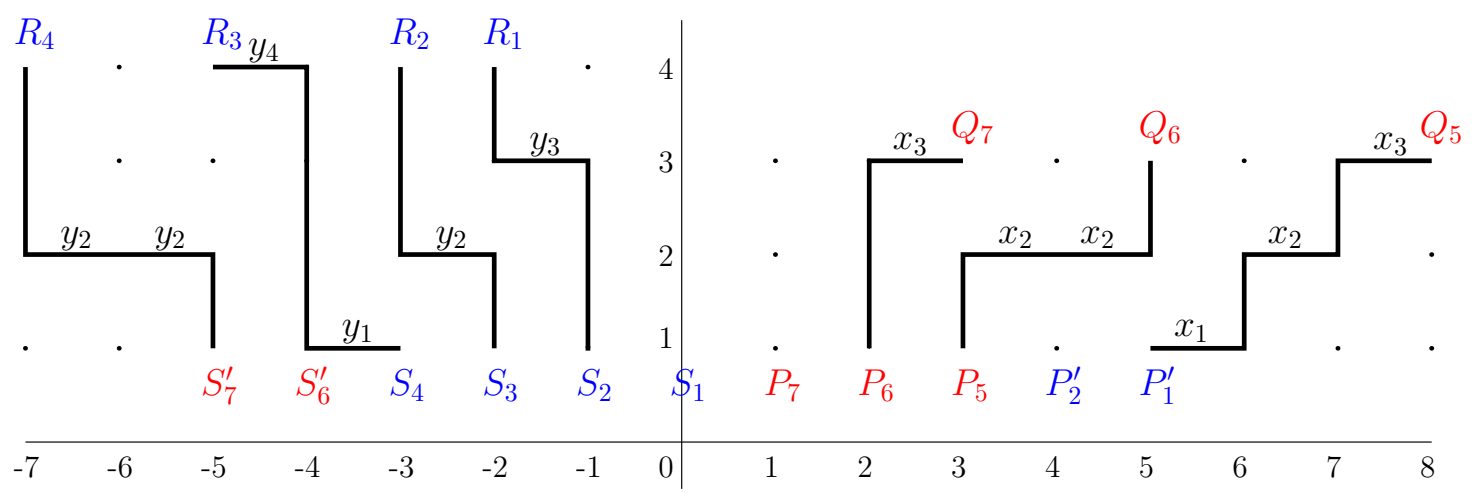

This owes its origin to the fact that $\pi$ specifies both the pairings of the end points of the paths:

$$
\left(\begin{array}{ccccccc}
R_{1} & R_{2} & R_{3} & R_{4} & Q_{5} & Q_{6} & Q_{7} \\
S_{2} & S_{3} & S_{4} & S_{7}^{\prime} & P_{1}^{\prime} & P_{5} & P_{6}
\end{array}\right)
$$

and the positions of the corresponding boldface elements $\mathbf{h}_{i}(\mathbf{x})$ and $\mathbf{h}_{j}(\mathbf{y})$ in the determinant:

$$
\left|\begin{array}{cccccccc}
h_{2}(\mathbf{y}) & \mathbf{h}_{\mathbf{1}}(\mathbf{y}) & 1 & 0 & \vdots & 0 & 0 & 0 \\
h_{3}(\mathbf{y}) & h_{2}(\mathbf{y}) & \mathbf{h}_{\mathbf{1}}(\mathbf{y}) & 1 & \vdots & 0 & 0 & 0 \\
h_{5}(\mathbf{y}) & h_{4}(\mathbf{y}) & h_{3}(\mathbf{y}) & \mathbf{h}_{\mathbf{2}}(\mathbf{y}) & \vdots & 0 & v h_{1}(\mathbf{y}) & v \\
h_{7}(\mathbf{y}) & h_{6}(\mathbf{y}) & h_{5}(\mathbf{y}) & h_{4}(\mathbf{y}) & \vdots & 0 & v h_{3}(\mathbf{y}) & v \mathbf{h}_{\mathbf{2}}(\mathbf{y}) \\
\cdots & \cdots & \cdots & \cdots & & \cdots & \cdots & \cdots \\
u \mathbf{h}_{\mathbf{3}}(\mathbf{x}) & u h_{4}(\mathbf{x}) & 0 & 0 & \vdots & h_{5}(\mathbf{x}) & h_{6}(\mathbf{x}) & h_{7}(\mathbf{x}) \\
u & u h_{1}(\mathbf{x}) & 0 & 0 & \vdots & \mathbf{h}_{\mathbf{2}}(\mathbf{x}) & h_{3}(\mathbf{x}) & h_{4}(\mathbf{x}) \\
0 & 0 & 0 & 0 & \vdots & 1 & \mathbf{h}_{\mathbf{1}}(\mathbf{x}) & h_{2}(\mathbf{x})
\end{array}\right| .
$$

The subscripts $i$ and $j$ of $\mathbf{h}_{i}(\mathbf{x})$ and $\mathbf{h}_{j}(\mathbf{y})$ determine the number of horizontal steps east and west, respectively, of the corresponding lattice paths. The north-east paths $P_{\pi(i)} Q_{i}$ and $P_{\pi(i)}^{\prime} Q_{i}$ contribute to $h_{\lambda_{i-n}-i+\pi(i)-c_{\pi(i)}}(\mathbf{x})$ with $c_{\pi(i)}=0$ and $p$, respectively, while the north-west paths $S_{\pi(i)} R_{i}$ and $S_{\pi(i)}^{\prime} R_{i}$ contribute to $h_{\mu_{m+n+1-i}+i-\pi(i)-d_{\pi(i)}}(\mathbf{y})$ with $d_{\pi(i)}=0$ and $q$, respectively.

In this particular example the chosen paths are non-intersecting. More generally, even for the same $\pi$ some of the paths contributing monomials to $\mathbf{h}_{i}(\mathbf{x})$ and $\mathbf{h}_{j}(\mathbf{y})$ will intersect. However, these will be cancelled by means of the usual sign changing involution that removes contributions from all intersecting paths. 
For example, consider the following $(n, m)$-tuple exhibiting an intersection of lattice paths:

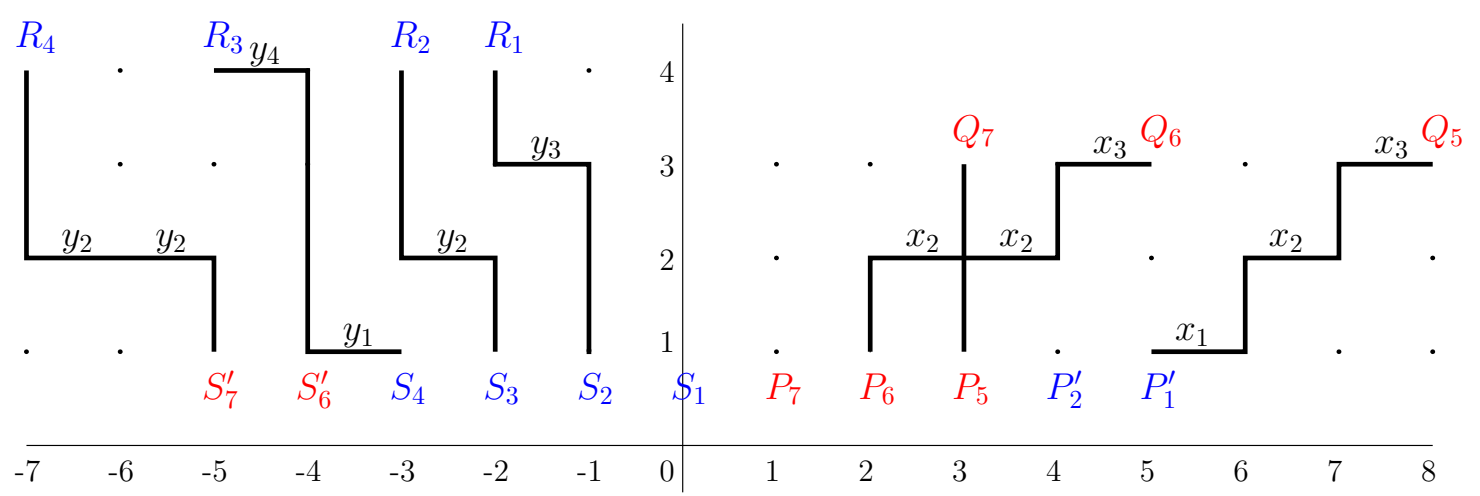

The sign changing involution, which may be identified in general from the left-most pair of intersecting paths, is provided in this case by the transposition $(5,6)$. The $(n, m)$-tuple contributes mutually cancelling monomials associated with the two permutations

$$
\left(\begin{array}{lllllll}
1 & 2 & 3 & 4 & 5 & 6 & 7 \\
2 & 3 & 4 & 7 & 1 & 5 & 6
\end{array}\right) \text { and }\left(\begin{array}{lllllll}
1 & 2 & 3 & 4 & 5 & 6 & 7 \\
2 & 3 & 4 & 7 & 1 & 6 & 5
\end{array}\right)
$$

where these two permutations, differing only by the transposition $(5,6)$, have parities \pm 1 .

Of course the north-east and north-west paths never intersect one another. In order to ensure that an $(n, m)$-tuple consists wholly of non-intersecting paths it is necessary that the corresponding permutation $\pi$ satisfies the constraints:

$$
\pi(1)<\pi(2)<\cdots<\pi(n) \text { and } \pi(n+1)<\pi(n+2)<\cdots<\pi(n+m) .
$$

Each such permutation $\pi$ may be written in the form

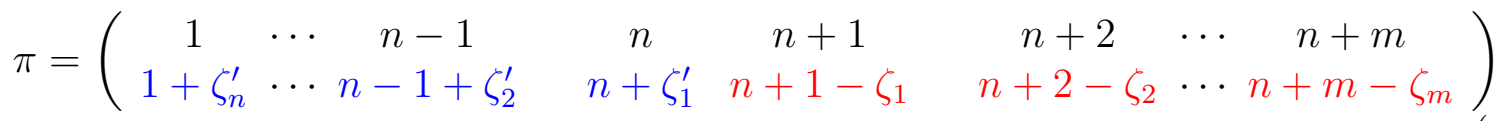

for some partition $\zeta \subseteq\left(n^{m}\right)$. Indeed, every such $\zeta \subseteq\left(n^{m}\right)$ arises in this way since there exists a bijective map from those permutations $\pi$ satisfying (44) to the partitions $\zeta \subseteq\left(n^{m}\right)$. This is constructed by labelling the consecutive boundary edges of $F^{\zeta} \subseteq F^{\left(n^{m}\right)}$ with integers $j=1,2, \ldots, n+m$, with the edge labelled $j$ either horizontal or vertical according as $\pi^{-1}(j)$ is either $\leq n$ or $>n$, respectively. Moreover, the rank $r(\zeta)$ of $\zeta$ is the maximum $k$ such that $\pi(n+k)=n+k-\zeta_{k} \leq n$, or equivalently $\pi(n-k+1)=n-k+1+\zeta_{k}^{\prime}>n$, and by counting descents

$$
(-1)^{\pi}=(-1)^{\zeta_{n}^{\prime}+\cdots+\zeta_{2}^{\prime}+\zeta_{1}^{\prime}}=(-1)^{|\zeta|} .
$$

By way of illustration, in the case of our example (39) for which

$$
\pi=\left(\begin{array}{lllllll}
1 & 2 & 3 & 4 & 5 & 6 & 7 \\
2 & 3 & 4 & 7 & 1 & 5 & 6
\end{array}\right)
$$


the differences in the entries in each column give $\zeta=(4,1,1)$ and $\zeta^{\prime}=(3,1,1,1)$, with $r=r(\zeta)=1$.

Quite generally, using the $\zeta$ and $\zeta^{\prime}$ obtained in this way, each $(n, m)$-tuple of nonintersecting lattice paths defines a pair of skew semistandard tableaux of shapes $\lambda / \sigma$ and $\mu / \tau$ with $\sigma=\left(\zeta+p^{r}\right)$ and $\tau=\left(\zeta^{\prime}+q^{r}\right)$. To be precise each $\sigma_{i}$ is the horizontal distance from $P_{n+i}$ to $P_{\pi(n+i)}^{\prime}$ for $i=1, \ldots, r$ and to $P_{\pi(n+i)}$ for $i=r+1, \ldots, m$, and for each particular $(n, m)$-tuple of non-intersecting lattice paths the entries in the $i$ th row of the skew semistandard tableau of shape $\lambda / \sigma$ are given by the consecutive heights $k$ of the horizontal steps of the lattice path from $P_{\pi(n+i)}^{\prime}$ or $P_{\pi(n+i)}$, as appropriate, to $Q_{n+i}$ for $i=1, \ldots, m$. Similarly, $\tau_{i}$ is the horizontal distance from $S_{n-i+1}$ to $S_{\pi(n-i+1)}^{\prime}$ for $i=1, \ldots, r$ and to $S_{\pi(n-i+1)}$ for $i=r+1, \ldots, n$, and the entries in the $i$ th row of the skew semistandard tableau of shape $\mu / \tau$ are given by the consecutive heights $k$ of the horizontal steps of the lattice path from $S_{\pi(n-i+1)}^{\prime}$ or $S_{\pi(n-i+1)}$, as appropriate, to $R_{n-i+1}$ for $i=1, \ldots, n$.

The fact that $s_{\lambda / \sigma}(\mathbf{x})$ and $s_{\mu / \tau}(\mathbf{y})$ can be defined by means of such skew semistandard tableaux then completes the combinatorial proof of (33).

QED

In the case $m=3, n=4, p=-2, q=-1, \lambda=(5,3,2)$ and $\mu=(4,3,2,2)$, our non-intersecting lattice path example, for which $\zeta=(4,1,1), \zeta^{\prime}=(3,1,1,1)$ and $r=1$, is such that the above is illustrated for the north-east paths by:

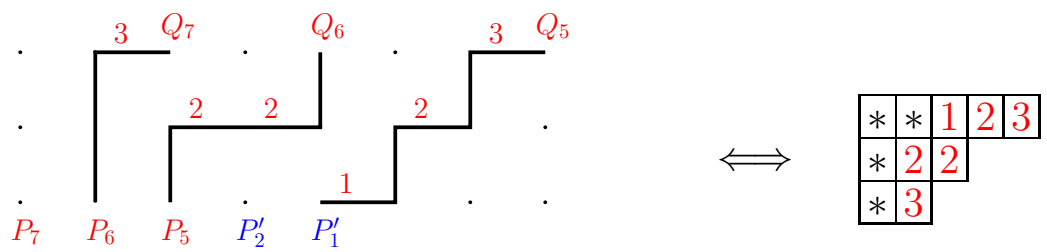

with

$$
\sigma=(2,1,1)=\left(\begin{array}{l}
1 \\
2
\end{array}\right)=\left(\begin{array}{c}
3-2 \\
2
\end{array}\right)=(4,1,1)+(-2,0,0)=\left(\zeta+p^{r}\right),
$$

and for the north-west paths by

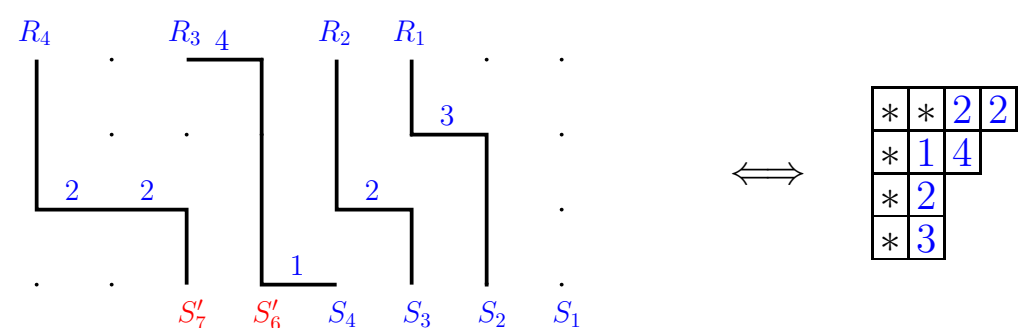

with

$$
\tau=(2,1,1,1)=\left(\begin{array}{l}
1 \\
3
\end{array}\right)=\left(\begin{array}{c}
2-1 \\
3
\end{array}\right)=(3,1,1,1)+(-1,0,0,0)=\left(\zeta^{\prime}+q^{r}\right) .
$$


Acknowledgements: The first author (AMH) acknowledges the support of a Discovery Grant from the Natural Sciences and Engineering Research Council of Canada (NSERC). The second (RCK) is grateful for the hospitality extended to him while visiting Wilfrid Laurier University, and for the financial support making such visits possible.

\section{References}

[1] D.M. Bressoud and S.Y. Wei, "Determinental formulae for complete symmetric functions", J. Comb. Theory A. 60 (1992) 277-286.

[2] A.M. Garsia, "On the maj and inv q-analogues of Eulerian polynomials", Lin. Multilin. Alg. 8 (1979), 21-34.

[3] I.M. Gessel and X. Viennot, "Binomial determinants, paths, and hook length formulae", Adv. Math 58 (1985), 300-321.

[4] I.M. Gessel and X. Viennot, "Determinants, paths, and plane partitions", preprint 1989 ; available at http://people.brandeis.edu/ gessel/homepage/papers/pp.pdf

[5] A.M. Hamel and R.C. King, "Extended Bressoud-Wei and Koike skew Schur function identities", to be published J. Comb. Theory A.

[6] S. Karlin and J.L. McGregor, "Coincidence properties of birth-and-death processes", Pacific J. Math 9 (1959), 1109-1140.

[7] S. Karlin and J.L. McGregor, "Coincidence probabilities", Pacific J. Math. 9 (1959), 1141-1164.

[8] K. Koike, "On the decomposition of tensor products of the representations of the classical groups: by means of the universal characters", Adv. Math. 74 (1989), 5786.

[9] B. Lindström, "On the vector representations of induced matroids", Bull. London Math. Soc. 5 (1973), 85-90.

[10] I.G. Macdonald, Symmetric Functions and Hall Polynomials, 2nd. Ed. Oxford: Oxford Univerity Press, 1995.

[11] R.P. Stanley, Enumerative Combinatorics, Volume 2, Cambridge: Cambridge University Press, 1999.

[12] J.R. Stembridge, "Nonintersecting paths, pfaffians, and plane partitions", Adv. Math. 83 (1990), 96-131. 